\title{
Autoimmune Diseases and Inflammatory Disorders of Unknown Etiology; Contemplating Causation and Implication in Public Health Protection
}

\author{
Ikonomopoulos John* \\ Faculty of Animal Science and Aquaculture, Agricultural University of Athens, Greece
}

Submission: January 14, 2017; Published: May 07, 2018

*Corresponding author: Faculty of Animal Science and Aquaculture ,Agricultural University of Athens, Iera Odos 75, Votanikos, Athens, Greece, Tel: 0030-6945484027; Fax: 0030-2105294388; Email: ikonomop@aua.gr

\begin{abstract}
Due to their multi factorial causation, the investigation of the etiology of autoimmune and inflammatory disorders constitutes a challenge that seems to exceed greatly that which was faced by the pioneers of the previous centuries, in connection with microbial diseases. Genetic factors, microbial pathogens, the alteration of the normal microbial flora and/or of the micro biome have been implicated to the etiology of the specific category of diseases but their interaction is so complex that the distinction of the causal from those that are incidental becomes extremely difficult. This issue is further complicated by food additives that are nowadays widely used and could have a contributing role to the pathogenesis of disorders associated with immune dysregulation. In this respect, it is perhaps critical that administrative authorities involved in public health protection revised their licensing strategy from deciding based on evidences of harmfulness, to taking seriously into consideration even indications of the latter or requiring proof of safety.
\end{abstract}

Keywords: Autoimmune diseases; Inflammatory disorders; Causation; Etiology; Erythritol; Arabinogalactan; Mycobacteria; Paratuberculosis; Crohn's disease

Abbreviations: ARPs: Antigen Recognition Patterns

\section{Introduction}

In ancient Greece, the notion of infectious agents was depicted with the term "miasma" that was used to indicate any factor resulting to bodily, spiritual or ethical deviation from normal. In 1665, Robert Hooke published his book titled "Micrographia", in which he provided a detailed description of the structure of a simple microscope that was invented many years before (1590), by Zacharias Janssen. This was an event of fundamental significance in the investigation of disease etiology and motivated several inspired researchers, such as Anton van Leeuwenhoek who provided the first physical proof of microscopic organisms in 1670, and Robert Koch with his landmark publication of the Koch's postulates in 1890. The latter constituted a concrete context that formulated the research conducted on all aspects of the investigation of disease etiology, for several decades. The idea was simple and common for all infectious diseases: a pathogen transmitted to a sensitive host causes a specific disease; the pathogen should not be detected in healthy individuals; a sensitive host exposed to the pathogen should develop the specific disease. These postulates proved efficient in connection with a large number of infectious diseases and triggered the developments towards their accurate diagnosis, treatment, control and in several cases, eradication.

However how does the scientific community respond today to the challenge represented by the dramatic rise in autoimmune and inflammatory disorders? If the elementary technological developments of the $17^{\text {th }}$ century proved sufficient to clarify disease pathogenesis of so many infectious diseases in just over a century, how is it possible that in spite the revolutionary discoveries of the $20^{\text {th }}$ century, a large number of wide spread diseases continues to be of unknown etiology? Is public health protection a parameter that should be considered in connection with the specific diseases or at least with those that appear to be potentially infectious, i.e. associated in many, but not all cases with exposure to one or more pathogens?

In contemplating the causation of autoimmune diseases and inflammatory disorders of unknown etiology, the logical approach would be to follow a course similar with that of Koch, and attempt to define first the notions of "disease" and "sensitive host"; exactly therein lies today the real challenge. Regarding case definition, what appears to be a single disease entity often 
proves to have specific forms that are similar in terms of clinical manifestation but different with regards to etiology. A typical example of the latter that was not as obvious a few years ago is cancer, a pathologic condition that is no longer perceived as a single disease and can be manifested by many similar forms, triggered by etiologic factors that are remarkably different such as viruses, chemicals, radiation etc. With regards to the definition of the sensitive host, the difficulty lies in that susceptibility does not apply at, but within the level of animal species, depending on various parameters including age, gender, race and genetic predisposition. The latter is neither precise nor definite i.e. there are many different factors possibly resulting to similar types of genetic predisposition and this can be influenced in the course of life by various parameters including stress, nutrition, alteration of the constitution of normal microbial flora, genetic and epigenetic factors. An example of a disease that seems to fit perfectly in the specific context of pathogenesis is Crohn's disease.

Crohn's disease is a form of chronic eleocolitis that affects humans with substantial negative impact to their quality of life [1]. Though the disease is still officially considered of unknown etiology, it is rather clear that the basis of its pathogenesis is immune dysregulation. Several factors have been proposed as relevant, such as genetic predisposition, nutrition, stress, the alteration of the microbial flora and/or of the microbiome, and various microbial organisms [2]. In connection with the latter, reference is more commonly made to Mycobacterium avium subsp. paratuberculosis (MAP) [2]. Notably MAP, which is wide spread in nature and many types of food, is the cause of paratuberculosis that affects the intestine primarily of ruminants, causing lesions that are very similar with those of Crohn's disease. It has been suggested that the implication of mycobacteria in this and other diseases of unknown etiology, such as sarcoidosis, is associated with the regulatory role they acquired in the process of the evolutionary development of the mammalian immune system, formulating its response to various antigen recognition patterns (ARPs), and its ability to selectively react or tolerate similar antigenic stimuli $[3,4]$.

This idea is the foundation based on which, a new approach was made very recently for the treatment of Crohn's disease patients, using a vaccine that contains MAP [5]. However, none of the factors mentioned above can be associated with all cases of disease, and causal effect cannot be determined in a definite manner for any of them, not even for a specific subset of patients. This has generated speculations about disease definition implying that maybe, just like cancer, Crohn's disease is a label that should be perceived as descriptive of a set of conditions that are similar with regards to clinical manifestation but distinct, in terms of etiology [2]. Unfortunately the issue of Crohn's disease causation is still very far from being resolved, mainly because the aforementioned factors are so closely interlinked that it becomes extremely difficult to differentiate the causal from those that are incidental. This problem however and the context of disease pathogenesis outlined here does not apply only to Crohn's disease. Some or all the factors associated with the causation of the specific pathologic condition are being identified as potentially significant for many others. To mention only a few, sarcoidosis has been associated through several reports with exposure of genetically predisposed individuals to Propionibacterium acnes or certain Mycobacterium spp. [6]; human exposure to MAP has been associated in addition to Crohn's disease with multiple sclerosis, diabetes mellitus type I, Hashimoto's thyreoiditis, sarcoidosis, rheumatoid arthritis and certain forms of cancer [2]; the alteration of the constitution of microbial flora and/or of the microbiome has been linked to a plethora of diseases of unknown etiology [4], the most recent and rather unexpected addition to which is autism [6].

The overall context formulated today in connection with diseases of unknown etiology would be incomplete without a reference to the potential role of specific food additives, which complicates even further the investigation of their pathogenesis. Two of the most widely used products of this category that could be implicated in the issue addressed here is erythritol and arabinogalactan. The former is a polyol that exists in a natural form in some fruits and vegetables but is used in an artificial form as a low calorie food sweetener, since it is very poorly metabolized by the intestinal and the oral microbial flora. Arabinogalactan is a polysaccharide consisting of arabinose and galactose, and can be found widely in nature, in plants and in the cell wall of specific bacteria. Today, arabinogalactan is used broadly as a chemical stabilizer in cosmetics, food and animal feeds, and as an immune stimulating or drug delivery agent in therapeutics and food supplements. Apparently due to the wide use of erythritol and arabinogalactan, human exposure to them exceeds greatly the natural, in terms both of intensity and duration. This could constitute an element of key significance in their role as potential or perhaps incidental pathogenic factors for specific sub-sets of the human population who, for various reasons, are prone to immune dysregulation. This association should be primarily assessed within the context of the microbial flora and secondarily in connection with the immune response to ARPs that the mammalian immune system has been trained to perceive as critical in terms of immune regulation and responsiveness. Indeed regarding the former, chronic exposure to erythritol is associated in many research reports with functional alteration of the intestinal microbiome, which may affect millions of consumers leading to autoimmune diseases and inflammatory disorders $[4,7,8]$. Erythritol is also a significant bioactive substance for potent bacterial pathogens. In more detail, the specific inert under normal circumstances sugar is identified as the factor that attracts Brucella abortus to the genital tract of female bovines, leading to chronic infection and abortion [9]. Erythritol has also been associated with up regulation of specific pathogenic genes of other Brucella spp., including Brucella melitensis, the main causal agent of brucellosis of humans [10]. Arabinogalactan on the other hand 
is a major component of the cell wall mainly of mycobacteria, nocardia and corynebacteria, and contributes significantly to their ability to resist phagocytosis and to survive as intracellular pathogens. In this respect the use of arabinogalactan as an immune stimulating agent seems rather apparent at least for normal individuals, but generates concern in connection with the response that it could activate to those prone to immune dysregulation, especially considering the strong indications about the implication of mycobacteria in autoimmune diseases and inflammatory disorders $[2,3,4,6]$.

\section{Conclusion}

The multi factorial causation of autoimmune diseases and inflammatory disorders that currently remain of unknown etiology constitutes a challenge that seems to exceed greatly that which was faced by the pioneers of the previous centuries, in connection with microbial diseases. The progress is indeed remarkable but there are no indications that the etiology of the specific pathologic conditions will be clarified in the near future. In this respect, it is nowadays perhaps critical that administrative authorities involved in public health protection revised their licensing strategy from deciding based on evidences of harmfulness, to taking seriously into consideration even indications of the latter or requiring proof of safety.

\section{References}

1. Ghosh S, Mitchell R (2007) Impact of inflammatory bowel disease on quality of life: Results of the European Federation of Crohn's and

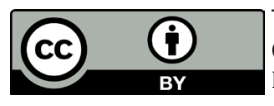

This work is licensed under Creative Commons Attribution 4.0 License DOI: 10.19080/IJCSMB.2018.04.555642
Ulcerative Colitis Associations (EFCCA) patient survey. J Crohns Colitis 1(1): $10-20$

2. Chiodini RJ, Chamberlin WM, Sarosiek J, McCallum RW (2012) Crohn's disease and the mycobacterioses: a quarter century later. Causation or simple association. Crit Rev Micro biol 38(1): 52-93.

3. Naser SA, Sagramsingh SR, Naser AS, Thanigachalam S (2014) Mycobacterium avium subspecies paratuberculosis causes Crohn's disease in some inflammatory bowel disease patients. World J Gastroenterol 20(23): 7403-7415.

4. Belkaid Y, Hand T (2015) Role of the Microbiota in Immunity and inflammation. Cell 157(1): 121-141.

5. http://www.crohnsmapvaccine.com

6. Saidha S, Sotirchos ES, Eckstein C (2012) Etiology of Sarcoidosis: Does Infection Play a Role. Yale J Biol Med 85(1): 133-141.

7. Mulle JG, Sharp WG, Cubells JF (2013) The Gut Microbiome: A New Frontier in Autism Research. Curr Psychiatry Rep 15(2): 337.

8. Suez J, Korem T, Zilberman-Schapira G, Segal E, Elinav E, et al. (2015) Non-caloric artificial sweeteners and the microbiome: findings and challenges. Gut Microbes 6(2): 149-155.

9. de Fiqueiredo P, Ficht TA, Rice-Fitcht A, Rossetti CA, Adams LG (2015) Pathogenesis and immunology of brucellosis: review of Brucella-host interactions. Am J Pathol 185(6): 1505-1517.

10. Petersen E, Raja shekara G, Sanakkayala N, Eskra L, Harms J, et al. (2013) Erythritol triggers expression of virulence traits in Brucella melitensis. Microbes Infect 15(6-7): 440-449.

Your next submission with Juniper Publishers will reach you the below assets

- Quality Editorial service

- Swift Peer Review

- Reprints availability

- E-prints Service

- Manuscript Podcast for convenient understanding

- Global attainment for your research

- Manuscript accessibility in different formats

( Pdf, E-pub, Full Text, Audio)

- Unceasing customer service

Track the below URL for one-step submission https://juniperpublishers.com/online-submission.php 